\title{
LA MASA NO SE CREA NI SE DESTRUYE. ¿ESTÁIS SEGUROS?
}

\author{
OÑORBE DE TORRE, A. ${ }^{1}$ y SÁNCHEZ JIMÉNEZ, J.M. ${ }^{2}$ \\ ${ }^{1}$ I.B. Fmilia Pardo Bazán, Madrid. \\ ${ }^{2}$ Universidad de Alcalá. Departamento de Química Física.
}

(Este trabajo es parte de una investigación más amplia realizada con la ayuda del CIDE. Ministerio de Educación y Ciencia.)

\section{SUMMARY}

From the very outset of the study of chemistry, the concepts of mass, physical and chemical transformations of matter and the law of conservation are fundamental. However, in reality, the learning of these concepts that pupils believe they understand is not very secure. When they try to apply their ideas to simple questions they make mistakes and the reasoning they use does not correspond to the explanations or definitions that they are able to repeat.

Los conceptos de masa y volumen y la ley más general de la química, la ley de la conservación de la masa, son conocimientos básicos que han de utitizarse, sin hacerlo explícitamente, en la gran mayoría de cuestiones y problemas. La ley de conservación de la masa es considerada generalmente, por profesores y alumnos, muy sencilla y fácilmente comprensible porque no conlleva un aparato matemático a su alrededor y porque su definición es fácilmente memorizable. Por tanto, su utilización no se analiza, se supone correcta en cualquier problema químico. Sin embargo, su aplicación supone una comprensión enlazada de otros muchos conceptos fundamentales como sustancia, materia, masa, volumen, densidad, etc.

"Conocer una definición, poder exponerla correctamente, no supone entender un concepto.» (Edwards y Mercer 1987). Puede ser una definicion memorística, repetitiva o comprendida a menor nivel de abstracción que el necesario para una determinada aplicación. Tambiért es posible que el fallo no se encuentre en la comprensión de conceptos aislados sino en la conexión entre ellos.

En este trabajo se pretende analizar si los alumnos creen comprender y son capaces de definir el concepto de masa y su ley de conservación relacionándolo posteriormente con la aplicación de estos conocimientos a la resolución de cuestiones directas, que no hacen necesaria la utilización de estrategias. La diferencia fundamental con otras investigaciones de objetivos o conclusiones simi- lares (Driver I985, Furió 1987, Séré 1986, Stävy 1991) se encuentra en el hecho de que los sujetos a los que se plantean las cuestiones-problema han respondido ya a la ley que debían aplicar y sería lógico suponer que, si la conocían y creían comprenderla, la utilizaran en sus respuestas.

\section{HIPÓTESIS PLANTEADAS}

- Los alumnos desde los 13-14 años creen tener claros los conceptos de masa y su conservación, cuando ya los han estudiado, especialmente la ley de conservación por su, aparentemente sencilla, definición.

- Los alumnos conocen algún tipo de explicación o definición del concepto de masa y de su ley de conservación. Son capaces de repetirlos aun cuando pueda hacer tiempo que no los han «estudiado».

- Parece lógico esperar que aquellos sujetos que definen y creen comprender unos conceptos puedan aplicarlos mayoritariamente a cuestiones sencillas. Sin embargo, suponemos que la convicción de entender la ley de conservación de la masa y la definición correcta de la misma no están relacionadas con el razonamiento utilizado en la resolución de problemas elementales que conllevan conservación de la masa. 


\section{PREPARACIÓN DE LOS CUESTIONARIOS Y OBTENCION DE DATOS}

Se prepararon diversas pruebas (Anexos I y II) que fueron utilizados previamente con una muestra de 74 sujetos entre 12 y 18 años (Oñorbe y Sánchez 1987) y con otro grupo đe mayor eđad (Ryan, Sánchez y Oñorbe 1989). A partir de estos resultados se modificaron aquellos puntos que resultaron de más difícil comprensión tanto en su lenguaje como en su presentación. También se prepararon a partir de estas pruebas las plantillas de corrección con las clasificaciones de las respuestas aparecidas.

- En la primera parte se pregunta si creen haber estudiado y comprendido algunos conceptos de química. Entre ellos se encuentra la masa y la ley de conservación, cuyas definiciones o posible explicación se solicitan posteriormente.

- En las siguientes pruebas (1,2 y 3$)$ se plantean algunas cuestiones-problema relacionadas con transformaciones físicas y químicas de la materia.

La cuestión $n^{\circ} 1$ sigue un esquema similar de presentación a la propuesta de Johnstone y Mughol (1979).

La $n^{\circ} 2$ es idéntica a la utilizada por Driver (1984) sobre conservación de la cantiơad de materia en una dísolución, y ${\operatorname{la~} n^{n}}^{\mathrm{3}}$, sobre cambio químico (combustión) y conservación, se ha diseñado para este trabajo manteniendo una estructura de presentación muy similar a la anterior y basada en problemas semejantes al Proyecto de la Universidad de Leeds "Children's Learning in Science Project» (Clis 1987)

\section{MUES'TRA UTILIZADA}

El número de sujetos cuyas respuestas fueron consideradas válidas fue de 713 , con edades entre 13 y 19 años y distribuidos en cursos y centros diferentes.

Tabla I

\begin{tabular}{|l|c|c|}
\hline CURSO & N DE ALUMNOS & CENTROS \\
\hline 8 EGB & 138 & 3 \\
1. BUP & 104 & 2 \\
29 BUP & 154 & 2 \\
39 BUP (CIENCIAS) & 106 & 3 \\
FP 2 GRADO & 87 & 2 \\
COU (CIENCIAS) & 124 & 3 \\
TOTAL & 713 & - \\
\hline
\end{tabular}

\section{ANÁLISIS DE RESULTADOS}

En la tabla II se relacionan los porcentajes de alumnos que dicen haber estudiado la masa y que creen comprenderla.
Tabla II

\begin{tabular}{|l|c|ccc|}
\hline CURSO & SI ESTLDIO & \multicolumn{4}{|c|}{ CRFE COMPRENDFRLA (\%) } \\
& LA MASA (\%) & Sí & NO & 0 \\
\hline $8^{\circ} \mathrm{EGB}$ & 99 & 68 & 23 & 8 \\
$1^{\circ} \mathrm{BUP}$ & 99 & 55 & 45 & 0 \\
$2^{\circ} \mathrm{BUP}$ & 99 & 64 & 36 & 0 \\
$3^{2} \mathrm{BUP}$ & 99 & 77 & 23 & 0 \\
FP 2 GRADO & 100 & 82 & 18 & 0 \\
COU & 99 & 70 & 30 & 0 \\
\hline
\end{tabular}

0 : No responde

En su posterior definición se han considerado correctas las respuestas Ilamadas 1 (del tipo: medida de la cantidad de materia) y $2(\mathrm{~m}=\mathrm{v} d, \mathrm{~m}=\mathrm{F} / \mathrm{a})$. Los resultados aparecen en la tabla III y parte de ellos y de la tabla II se reflejan en la gráfica (Fig. 1).

Tabla III

Resultados porcentuales.

\begin{tabular}{|c|c|c|c|c|c|c|c|c|}
\hline CURSO & 1 & 2 & $1+2$ & 3 & 4 & 5 & 6 & 0 \\
\hline $8^{2} \mathrm{HGB}$ & Ll & 0 & 11 & 2 & 7 & 30 & 9 & 38 \\
\hline $1 \circ$ BUP & 42 & 8 & 50 & 7 & 7 & 18 & 5 & 13 \\
\hline $2^{\circ} \mathrm{BUP}$ & 24 & 8 & 32 & 3 & 10 & 31 & 6 & 18 \\
\hline $3^{n}$ BUP & 19 & 21 & 40 & 5 & 5 & 20 & 17 & 14 \\
\hline$F P$ & 21 & 8 & 24 & 8 & 1 & 26 & 20 & 17 \\
\hline $\mathrm{COU}$ & 36 & 6 & 43 & 5 & 1 & 16 & 15 & 23 \\
\hline \multicolumn{9}{|c|}{$\begin{array}{l}\text { I. Respuesta conrecta. } \\
\text { 2. Respuesta correcta mediante fórmula. } \\
\text { 3. Fórmula errónea. } \\
\text { 4. Confusión con volumen. } \\
\text { 5. Confusión con peso-gravedad. } \\
\text { 6. Otras } \\
0 . \text { No responde. }\end{array}$} \\
\hline
\end{tabular}

Figura 1

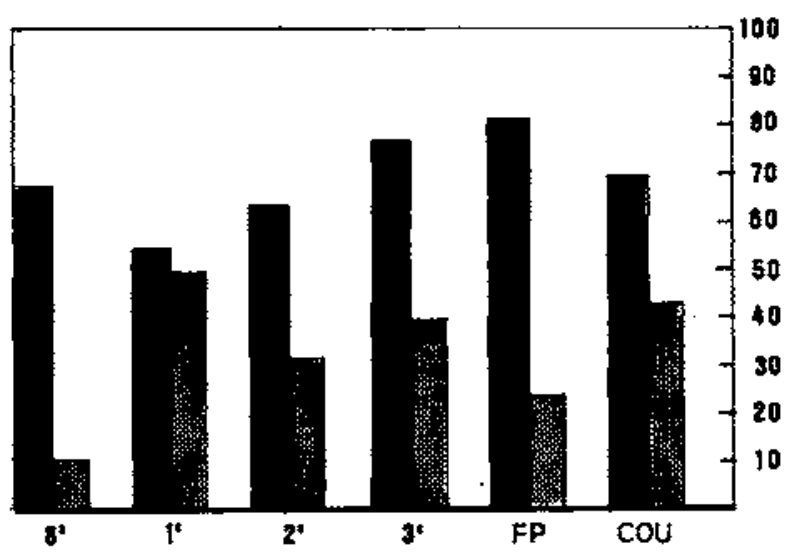

mCrosn conprascor 
En la pregunta sobre la ley de conservación de la masa un alto porcentaje contesta haberla estudiado. En su comprensión disminuye mucho este porcentaje especialmente en los grupos de $1^{2}$ y $2^{2}$ de BUP.

Tabla IV

\begin{tabular}{|c|c|c|c|c|c|c|}
\hline \multicolumn{4}{|c|}{ CURSO ESTUDIO LA LEY (\%) } & \multicolumn{3}{|c|}{ CREE COMPRENDERLA (\%) } \\
\hline & sf & $\mathrm{NO}$ & 0 & \$I & No & 0 \\
\hline $8^{\circ} \mathrm{HGB}$ & 73 & 22 & 5 & 41 & 44 & 15 \\
\hline $1^{\circ} \mathrm{BUP}$ & 69 & 31 & 0 & 19 & 81 & 0 \\
\hline $2^{8} \mathrm{BUP}$ & 56 & 55 & 0 & 10 & 90 & 0 \\
\hline $3^{2}$ BUP & 95 & 5 & 0 & 58 & 42 & 0 \\
\hline $\mathrm{HP}$ & 75 & 25 & 0 & 33 & 67 & 0 \\
\hline $\mathrm{COU}$ & 91 & 8 & 1 & 65 & 34 & 1 \\
\hline
\end{tabular}

En su definición se han considerado aceptables la conservación general -1- y la aplicada solo a reacción química -2-. La tabla $\mathrm{V}$ muestra los datos y la gráfica (Fig. 2) recoge parte de los resultados de las tablas IV y V.

Tabla V

Definición de la ley. (Rcsultados porcentuales).

\begin{tabular}{|c|c|c|c|c|c|c|c|}
\hline CURSO & 1 & 2 & $1+2$ & 3 & 4 & 5 & $-\underline{0}$ \\
\hline $8^{\circ} \mathrm{EGB}$ & 19 & 1 & 20 & 2 & 0 & 6 & 72 \\
\hline$I^{P}$ BUP & 20 & 1 & 21 & 3 & 0 & II & 65 \\
\hline $2^{2}$ BUP & 13 & 1 & 14 & 0 & 2 & 10 & 13 \\
\hline $3^{\circ}$ BUP & 46 & 10 & 57 & 0 & 0 & 13 & 30 \\
\hline $\mathrm{FP}$ & 28 & 1 & 29 & 3 & 2 & 18 & 47 \\
\hline $\mathrm{COU}$ & 44 & 14 & 59 & 0 & 1 & 8 & 33 \\
\hline \multicolumn{8}{|c|}{$\begin{array}{l}\text { 1. Correcta. } \\
\text { 2. Limitada a cambio químico. } \\
\text { 3. Confusión con energía. } \\
\text { 4. Aplicable solo cuando no hay reaccion. } \\
\text { 5. Otras. } \\
\text { 0. No responde. }\end{array}$} \\
\hline
\end{tabular}

Figura 2

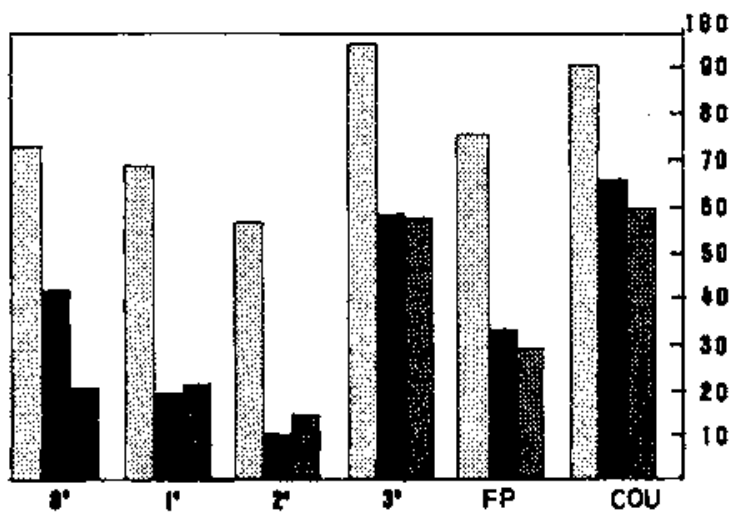

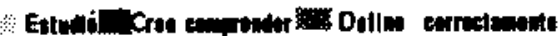

Io cuestión: Comparación de masas y volúmenes entre distintas sustancias y cambios en sus formas gcométricas.

Los porcentajes de respuestas correctas a las cuatro preguntas planteadas son muy bajos. La gráfica (Fig. 3) muestra estos resultados para cada una de las preguntas. Puede observarse que un máximo del $12 \%$ en alumnos de $3^{\circ}$ consigue responder correctamente al conjunto completo de cuestiones.

Figura 3

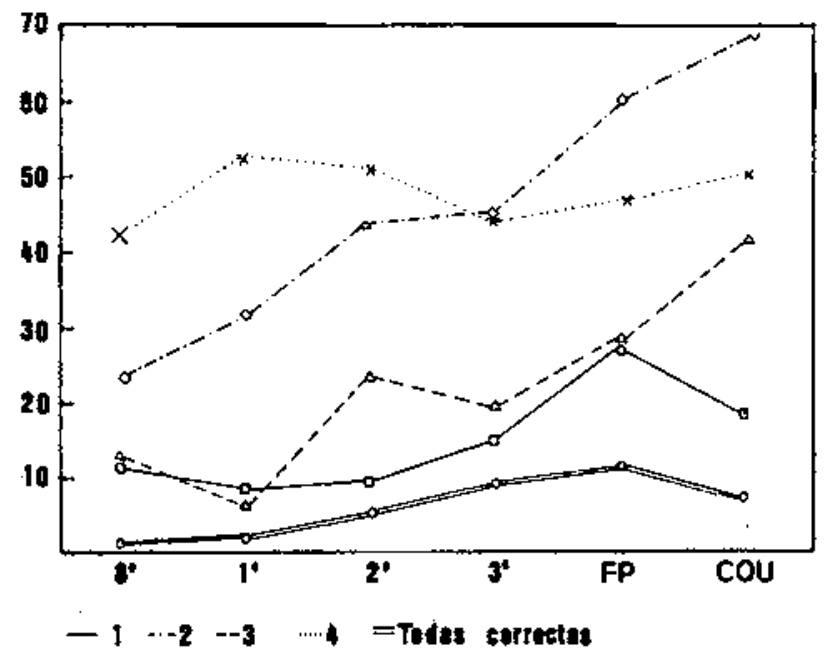

$2^{a}$ cuestión: Disolución.

El resultado correcto (1200 gramos) y su razonamiento adecuado son muy semejantes y aumenta con la edad hasta alcanzar el $75 \%$ en $3^{\circ}$ de BUP y COU. Entre los alumnos de 14,15 y 16 años no hay diferencias significativas. Las gráficas de las figuras 4 y 5 muestran los datos obtenidos.

Figura 4

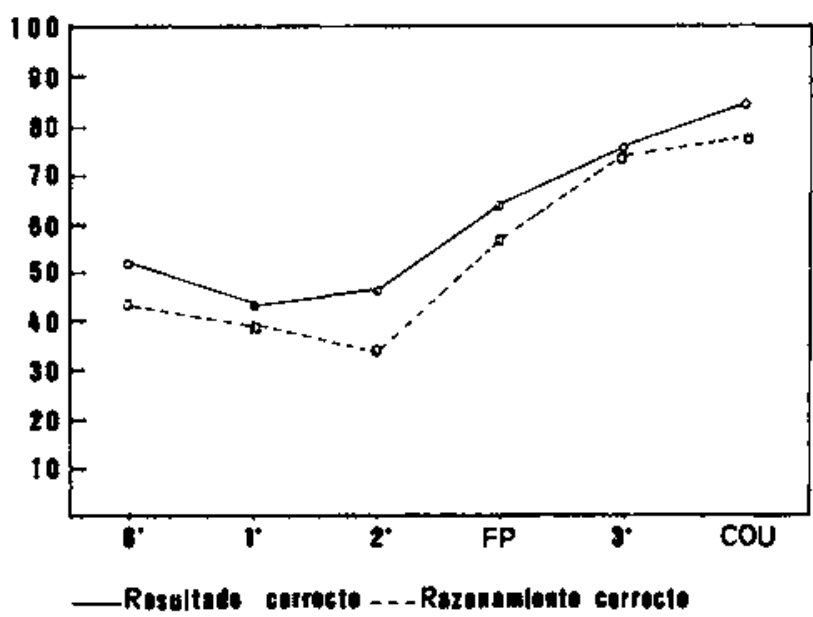


Figura 5

El azúcar desaparcce.

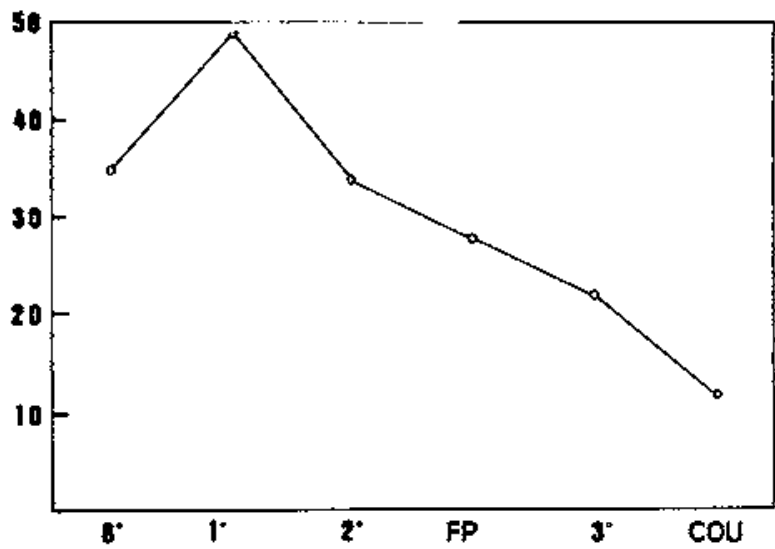

\section{$3^{4}$ Cuestion: Combustión.}

Las respuestas correctas son mucho menores que en la cuestión anterior, sólo alcanzan el $40 \%$ en el curso de COU cuando se tiene en cuenta también el razonamiento que utilizan. Las figuras 6 y 7 muestran gráficamente los resultados numéricos y de razonamiento.

Figura 6

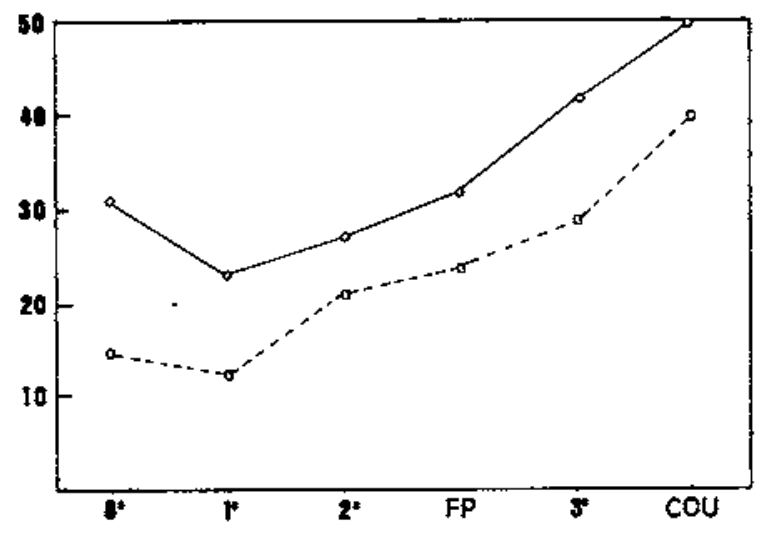

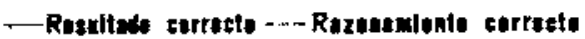

Al comparar los resultados correctos de las cuestiones sobre disolución y combustión puede observarse que los de esta última son, para todos los cursos, del orden de la mitad $o$ aún menores que en el problema de la disolución.

Para comprobar si existe correspondencia entre la definición de la ley y su utilización posterior en los problemas se han cruzado los datos correspondientes a:

A) Alumnos que definen la ley y razonan correctamente en la disolución.
Figura 7

Pêrdida de masa total.

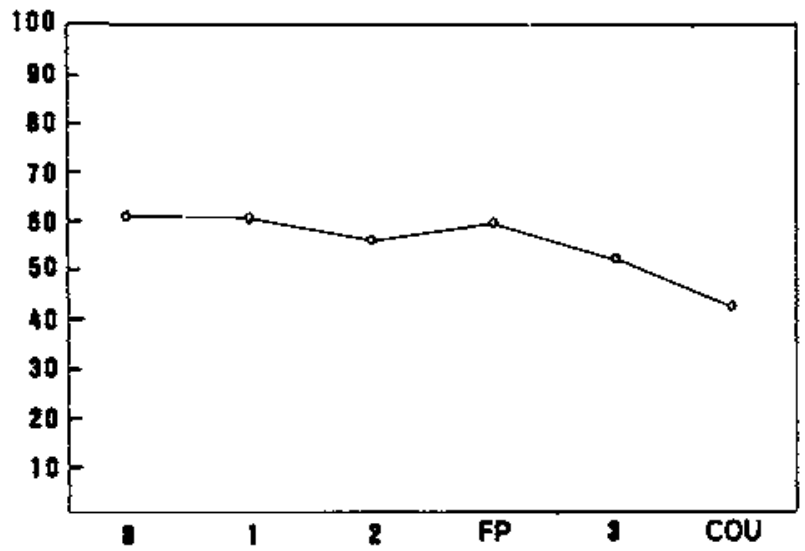

B) Alumnos que no definen la ley y razonan correctamente en la disolución.

C) Alumnos que definen la ley y razonan correctamente en la combustión.

D) Alumnos que no definen la ley y razonan correctamente en la combustión.

La tabla siguiente (Tabla VI), referida a los resultados de A), B), C) y D), muestra claramente que el hecho de definir correctamente la ley de conservación no está relacionado con su aplicación en los cambios de la materia.

Tabla VI

\begin{tabular}{|lcccc|}
\hline \multirow{2}{*}{ CURSO } & \multicolumn{2}{c}{ DISOLUCION (\%) } & \multicolumn{3}{c|}{ COMBUSTION (\%) } \\
& A & B & C & D \\
$8^{\circ}$ EGB & 6 & 37 & 5 & 10 \\
$I^{\circ}$ BUP & 11 & 29 & 0 & 8 \\
$2^{8}$ BUP & 5 & 30 & 1 & 4 \\
FP & 20 & 38 & 0 & 13 \\
$3^{\circ}$ BUP & 28 & 45 & 4 & 11 \\
COU & 35 & 42 & 10 & 18 \\
\hline
\end{tabular}

En eI análisis global de los resultados puede observarse que la contestación totalmente adecuada al conjunto de las pruebas es casi nula. No son los mismos sujetos los que responden correctamente a las diferentes cuestiones. Al analizar separadamente los distintos grupos de un mismo curso se encuentran resultados muy similares en las pruebas correspondientes a las cuestiones-problema. No ocurre lo mismo en las definiciones utilizadas para definir masa y ley de conservación. Los grupos de un mismo nivel pueden presentar gran diferencia en el número de alumnos que contestan o no correctamente. 
Esta variedad creemos que puede ser debido a la enseñanza recibida sin implicar, como se muestra en los razonamientos utilizados para explicar las cuestiones posteriores, una comprensión de aquello que están definiendo.

\section{CONCLUSIONES}

- Un alto porcentaje de alumnos, especialmente en los cursos superiores, cree comprender el concepto de masa y la ley de su conservación siendo capaz de definirlas en forma adecuada. Sin embargo es muy bajo el númejo de sujetos capaces de responder correctamente a todas las cuestiones planteadas sobre diversas transformaciones de la materia, puesto que conservan la masa en una determinada pregunta y no lo hacen en otra.

- No existe correlación entre la definición correcta de la ley y su utilización posterior. Es mayor el número de sujetos que sin definir la ley son capaces de razonar con ella en las distintas cuestiones que aquéllos que la definen y posteriormente la aplican.

- En las transformaciones presentadas como disolución de una sustancia y combustión, la corrección de respuestas y razonamientos crece con la edad el nivel de estudios científicos. Sin embargo es claro que la combustión, donde la transformación sufrida por la materia es mucho mayor, se admite con más facilidad la desaparición de la materia. El $60 \%$ de los alumnos que estaban a punto de concluir el Curso de Orientación Universitaria en la opción de ciencias no son capaces de razonar la conservación de la masa en una reacción química perfectamente conocida.

-El porcentaje de alumnos que responden correctamente a las diferentes pruebas y cuyo razonamiento es adecuado va creciendo con el nivel de estudios científicos y con la edad del que contesta. Los resultados en general son bajos $y$, aunque son acordes con otras investigaciones, la mayor edad de los sujetos encuestados por nosotros y el hecho de haber preguntado previamente la ley que debían utilizar no parece haber influido en los resultados finales. La definición correcta, memorizada, hace creer a muchos alumnos en su comprensión pero no ayuda al razonamiento posterior.Todo ello parece indicar un aprendizaje poco significativo aun en los cursos de mayor nivel de estudios de química.

En un análisis cualitativo de los razonamientos empleados en las diferentes pruebas se obtienen algunas conclusiones que interesa reseñar:
- Los alumnos de menor edad confunden masa con «algo que se toca», "se ve» o que "ocupa sitio». Aunque la palabra volumen no suele ser utilizada por cllos, son los que en mayor proporción lo confunden con la masa en los razonamientos utilizados para explicar las cuestiones de disolución y combustión.

- La utilización del peso y de la "gravedad de un cuerpo» como definición de masa aparece posteriormente en la medida que comienza el estudio de Física y Química más formalizado. Este error es el fundamental $(50 \%)$ en Ios alumnos de 2 . También, y a medida que aumenta el nivel de estudios, se utiliza más lenguaje matemático, correcto o no, en las definiciones de la masa de un cuerpo (del tipo $\mathrm{m}=\mathrm{P} / \mathrm{g} \circ \mathrm{m}=\mathrm{V}$.d).

- Respecto al volumen se ha detectado un porcentaje alto de confusión entre volumen y superficie en los alumnos de mayor edad cuando el número correspondiente a estas magnitudes es el mismo, sin considerar las unidades manejadas (es el caso de las figuras B y C de la primera cuestión). Como hipótesis se sugiere la tendencia, acentuada por la enseñanza recibida, a utilizar datos numéricos y cálculos como fuente de verdad independientemente del concepto implicado.

- En los alumnos de mayor nivel de estudios, se observa en sus razonamientos una mayor utilización (generalmente inadecuada) de términos que han estudiado y que consideran científicos: «Como no hay una reacción química los reactivos no se transforman y la masa no varía», "porque son dos elementos que forman un compuesto», «porque desaparece la masa convertida en energía según Einstein»...

- Los diversos errores cometidos y los razonamientos empleados por un mismo alumno no presentan coherencia sino que pueden variar según la cuestión planteada. Así, alumnos que diferencian aparentemente masa y volumen en una respuesta confunden luego ambos conceptos en otra, $O$ aquéllos que razonan que la materia no puede desaparecer al disolverse un cuerpo no encuentran dificultad para aceptar que disminuya en la combustión.

En resumen, todos los datos obtenidos y el análisis de los mismos vienen a indicar que el aprendizaje actual de algunos conceptos básicos para el estudio de la Química es puramente memorístico o al menos no significativo, puesto que no es después aplicable a cuestiones sencillas. Se hace necesario un replanteamiento de la enseñanza de estos fundamentos ya que sólo a partir de una verdadera comprensión de los mismos podrá accederse a otros conceptos químicos. Es de destacar también que la base de la resolución de un gran número de problemas en quimica es la aplicación explícita o implícita de la ley de conservación de la masa. 


\section{REFERENCIAS BIBLIOGRÁFICAS}

CLIS, 1987. Approaches to teaching the particles theory of matter. (Centre for Studies in Science and Mathematics Education. University of Leeds: Leeds).

DRIVER, R. y CHILD, D., 1984. Science in Schools. Age 15. Rescarch report 2. APU. (Department of Education and Science: Londres).

DRIVER, R., GUESNE, E. y TIBERGHIEN, A., 1989. Ideas científicas en la infancia y en la adolescencia. (Children's ideas in Science, 1985). (MEC y Morata: Madrid).

EDWARDS, D. y MERCER, N., 1987. The development of under-stonding in the classroom. (Londres).

FURIO, C.J., PEREZ, J.H. y HARRIS, H.H., 1987. ParalleIs between adolescents' conception of gases and the history of chemistry, Journal of Chemical Education, 64, pp. 616-618.

JOHNSTONE, A.H. y MUGHOI, A.R.N., 1979. Testing for under-standing, The School Science Review, 214(61), pp. $147-150$.

OÑORBE DE TORRE, A. Y SÁNCHEZ,JMÉNEZ, J.M., 1987. El concepto de masa en alumnos entre 12 y 18 años. Comunicación presentada en el // Congreso Internacional de Enseñanza de las Ciencias. Valencia.

RYAN, C., SÁNCHEZ, J.M. y OÑORBE, A., 1989. Scientific Ideas held by Interding primary Teachers in Britain and Spain, European Journal of Teacher Education, 12(3), pp. 239-251.

SERE, M. G., 1986. Children's conceptions of the gaseous state, prior to teaching, European Journal of Science Edu. cation, 8(4), pp. 413-425.

STAVY, R. 1991. Using Analogy to Overcome Misconceptions about Conservation of Matter, Journal of Research in Science Teaching, 28(4), pp. 305-313.
- Señala con una cruz en las columnas los conceptos que estudiaste alguna vez y los que crees comprender bien:

\section{Lo estudie Lo comprendo} alguna vez, bien.

1. Masa de un cuerpo

2. Volumen de un cuerpo

3. Densidad

4. Evaporación

5. Disolvente

6. Disolucion

7. Reacción química

8. Fcuación química

9. Ley de conservación de la materia

10. Combustion

11. Oxidación

12. Elemento

- Explica o define los señalados con los números 1 y 9 , y otro concepto cualquiera que elijas.

\section{PROBLEMA 1}

Observa las siguientes figuras y responde a las preguntas:

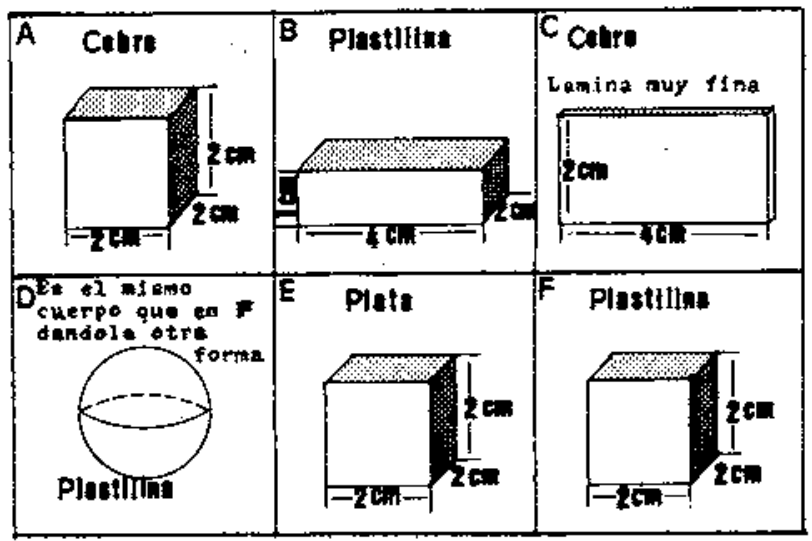

- A tiene igual volumen que

- B tiene igual masa que $\ldots, \ldots, \ldots, \ldots, \ldots$

- A tiene igual masa que

- B tiene mayor volumen que

Si alguna pregunta te resulta dudosa explica por qué. 


\section{PROBLEMA 2}

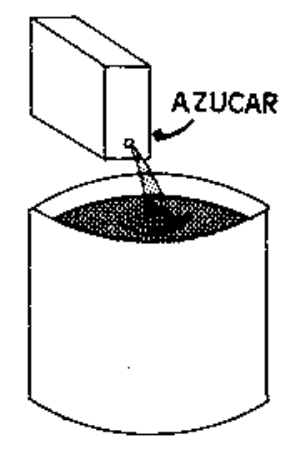

- En un cazo, como el de la figura, se añaden 200 gramos de azúcar a 1000 gramos de agua y se agita hasta que to 10 el azúcar se disnelve.

a) El contenido del cazo tendrá ahora una masa de (hacer una cruz en la casilla correspondiente):

- Menos de 1000 gramos.

- 1000 gramos.

- Más de 1000 gramos pero menos de 1200 gramos.

- 1200 gramos.

-. Más de 1200 gramos.

b) Explicar las razones por las que se ha elegido la respuesta.
PROBLEMA 3

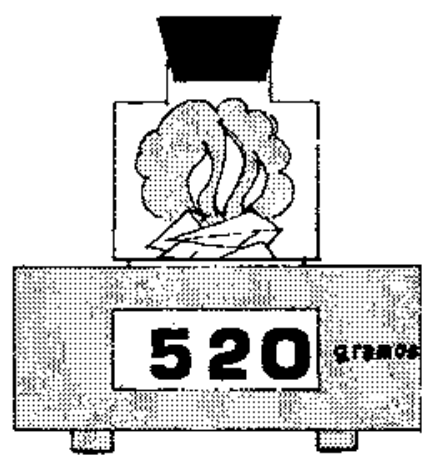

- En una botella, como la de la figura, situada sobre una baianza, se introduce un papel encendido que pesa 20 gramos y se cierra inmediatamente.

Una vez cerrada, la aguja de la balanza marca 520 gramos. Cuando el papel se queme totalmente la balanza marcará:

- Menos de 500 gramos.

-500 gramos.

- Entre 500 y 520 gramos.

-520 gramos.

- Más de 520 gramos.

Explicar las razones por las que se ha elegido la respuesta. 